\title{
Open System Model of Choice and Response Time
}

\author{
Gunnar P. Epping (gunnarepping@gmail.com) \\ Department of Psychological and Brain Sciences, 1101 E. 10th Street \\ Bloomington, IN 47405 USA
}

Peter D. Kvam (pkvam@ufl.edu)

Department of Psychology, 945 Center Dr.

Gainesville, FL 32603 USA

Timothy J. Pleskac (pleskac@ku.edu)

Department of Psychology, 1415 Jayhawk Blvd. Lawrence, KS 66045 USA

\author{
Jerome R. Busemeyer (jbusemey@indiana.edu) \\ Department of Psychological and Brain Sciences, 1101 E. 10th Street \\ Bloomington, IN 47405 USA
}

\begin{abstract}
Sequential sampling models have provided accurate accounts of people's choice, response time, and preference strength in value-based decision-making tasks. Conventionally, these models are developed as Markov-type processes (such as random walks or diffusion processes) following the Kolmogorov axioms. Quantum probability theory has been proposed as an alternative framework upon which to develop models of cognition, including quantum random walk models. When modeling people's behavior during decision-making tasks, previous work has demonstrated that both the Markov and quantum models have their respective strengths. Recently, the open system model, which is a hybrid version of the Markov and quantum models, has been shown to provide a more accurate account of preference strength compared to the Markov and quantum models in isolation. In this work, we extend the open system model to make predictions on pairwise choice and response time and compare it to the Markov and quantum random walk models.
\end{abstract}

Keywords: open system model; random walk model; decision-making; sequential sampling process; quantum cognition

\section{Introduction}

As an individual evaluates different alternatives leading up to a value-based decision (e.g., "What food would you like for lunch?"), popular models of decision-making often assume that preference accumulates over time for each alternative. Ultimately, once preference accumulates above a certain threshold for a given alternative, a decision is made, and that alternative is selected. The process of preference accumulation is commonly modeled as a sequential sampling process, and these models have accurately been used to predict individuals' choice, response time, and preference strength in value-based decision-making tasks (Busemeyer, Gluth, Rieskamp, \& Turner, 2019). Specifically, in a two-alternative forced-choice task (2AFC), this sequential sampling process is typically formalized as a Markov random walk on a one-dimensional lattice of preference states (Bhattacharya \& Waymire, 2009), where the two terminal states correspond to the two alternatives in the $2 \mathrm{AFC}$. Note, this process has also been formalized as a one-dimensional diffusion process (Ratcliff, Smith, Brown, \& McKoon, 2016), which is a continuous-state version of the Markov random walk process. As the decision-maker accumulates preference for a given alternative, the preference state shifts in the direction of the corresponding terminal state of that alternative. Assuming an internally controlled stopping procedure (i.e., the decisionmaker is free to respond once they've made a decision rather than being queued for a decision at a specific time), an alternative is selected when the preference state walks into one of the corresponding terminal states (referred to as an absorbing boundary random walk process).

As indicated by the name, random walk processes are inherently stochastic and therefore make probabilistic predictions about individuals' choice, response time, and preference strength. Probabilistic models must specify an underlying probability theory that dictates how the probability of events (measurement results) are generated from the model. This step in developing cognitive models is often overlooked and researchers tend to default to traditional probability theory as axiomatized by Kolmogorov (1950). Recently, quantum probability theory has been proposed as an alternative framework upon which to develop cognitive models, including random walk models (Busemeyer, Wang, \& Townsend, 2006), and has been successful in accounting for seemingly irrational behavior that pose challenges to traditional cognitive models (Bruza, Wang, \& Busemeyer, 2015).

\section{Conceptual Differences Between Markov and Quantum Random Walk Models}

The transition from traditional to quantum probability theory dramatically alters how uncertainty is incorporated into each random walk process. In a Markov random walk, the decision-maker's preference state is represented as a definite state; whereas in a quantum random walk, the decisionmaker's preference state is represented as a superposition state. 
To grasp the difference between these two representations, consider the probability of flipping a coin and it landing heads-up versus the probability of it landing tails-up. There's a $50 \%$ chance heads and a $50 \%$ chance of tails, right? Not exactly. If you were to know the exact state of the system (i.e., the position at which you release the coin, the velocity at which you flip it, the density of air in the room, etc.), you would be able to predict with $100 \%$ certainty which side would land up. Therefore, the uncertainty incorporated into our classical model of the coin flip is introduced due to our (the modeler's) lack of knowledge about the state of the system. This is a sort of epistemic uncertainty that accompanies modeling a system using a definite state (Busemeyer, Kvam, \& Pleskac, 2020).

To contrast the coin flip example with a quantum system, consider measuring whether the spin of an electron is spin up or spin down. Physicists have demonstrated that even if you have complete knowledge of the state of the system, there is still a $50 \%$ chance of measuring the spin of the electron to be spin up and a $50 \%$ chance of measuring it to be spin down (Hughes, 1989). Here, rather than uncertainty being incorporated as the result of our lack of knowledge of the system, uncertainty is an intrinsic property of the system. This is a sort of ontic uncertainty that accompanies modeling a system using a superposition state (Busemeyer, Kvam, \& Pleskac, 2020).

By using a definite state to model preference accumulation, a Markov random walk assumes that the decision-maker is explicitly aware of the location of their preference state along the one-dimensional lattice. When a decision is made, a person simply reads out their preference from a pre-existing definite state. This is similar to how once we flip a coin, observing which side landed up simply reveals the state of the coin, which we could have already known with $100 \%$ certainty if we had complete knowledge of the system. Therefore, the uncertainty included in the Markov random walk process is the modeler's lack of knowledge of the decision-maker's preference state.

By using a superposition state to model preference accumulation, a quantum random walk assumes that the decisionmaker is uncertain about the location of their own internal preference state along the one-dimensional lattice. When a decision is made, a person constructs a definite preference out of the superposition state. Therefore, the uncertainty included in the quantum random walk process is the decision-maker's own lack of knowledge of their preference state, rather than the modeler's lack of knowledge.

Although a definite state sounds appropriate for some decisions, such as if someone asks you your favorite color or favorite sports team, a superposition state appears more apt for other decisions, such as if someone asks you how you are feeling.

As indicated earlier, random walk models are often evaluated on their ability to account for choice, response time, and preference strength in a value-based decision-making task.
Wang and Busemeyer (2016) found that the quantum model provided a better fit compared to the Markov model when accounting for choice in a sequential decision-making task. But, when fit to pairwise choice and response time data, both Busemeyer et al. (2006) and Fuss and Navarro (2013) reported that the Markov random walk outperformed the quantum random walk. Finally, when fit to joint choice and confidence data (confidence is analogous to preference strength in an evidence-based decision-making task), Kvam, Pleskac, $\mathrm{Yu}$, and Busemeyer (2015) concluded that the quantum random walk outperformed the Markov random walk. Importantly, the quantum model can account for interference effects in joint choice and confidence data (i.e., making a choice alters the distribution of confidence ratings) which is not compatible with the Markov model. It appears that there are benefits that accompany both models. Up to this point we've discussed random walk models as either being purely Markov or purely quantum, but is necessary to treat Markov and quantum random walk models as mutually exclusive?

\section{Open System Model}

Fortunately, that is not the case. The open system random walk model combines quantum and Markov models in a hybrid fashion such that the quantum random walk and the Markov random walk are merely two ends of a random walk spectrum spanned by the open system model (Busemeyer, Zhang, Balakrishnan, \& Wang, 2020; Fuss \& Navarro, 2013; Martínez-Martínez \& Sánchez-Burillo, 2016). Being a hybrid version of the Markov and quantum models, the open system model incorporates both epistemic and ontic uncertainty into the random walk process, which is probably the most accurate representation as both a decision-maker is uncertain of the location of their evidence state and the modeler is also uncertain of the location of the decision-maker's superposition state (Yearsley, 2017).

When fit to preference strength data, Kvam, Busemeyer, and Pleskac (2021) reported that the open system model outperforms both Markov and quantum random walk models. Kvam et al. (2021) also demonstrated that mean preference strength systematically oscillates over time and that a decision causes an interference effect on the subsequent preference strength. Both of these findings are incompatible with the Markov model, which predicts a monotonic increase in mean preference strength and no interference effect, but are naturally accounted for by the open system and quantum models. In addition to comparing quantum and Markov models, Fuss and Navarro (2013) also fit an open system model to pairwise choice and response time data. They found that the open system model produced a small advantage over the Markov model. Although this is a promising result, the open system model used in Fuss and Navarro (2013) has little connection to previous Markov models of decision-making. This model was developed as an array of parallel accumulators, where each accumulator is assigned a phase. The phase difference between any two accumulators determines the degree to which those two accumulators reinforce each other. With 
this formulation, the open system model in Fuss and Navarro (2013) can be represented by a quantum random walk with a single accumulator, but their forms are quite different.

In this article, we develop a more general open system model to predict pairwise choice and response time data. This model builds off of previous Markov and quantum models of decision-making (Busemeyer, Zhang, et al., 2020) and also incorporates a parameter that estimates the relative influence of the Markov and quantum components on the random walk. Rather than assuming an array of accumulators like the model in Fuss and Navarro (2013), we assume a single accumulator. Also, the dynamics of the open system model outlined here are governed by the same tridiagonal intensity and Hamiltonian matrices that determine the dynamics of Markov and quantum random walks, respectively. After developing this more general open system model, we compare its performance to that of the Markov and quantum models when fit to pairwise choice and response time data.

\section{Methods}

\section{Participants}

A total of $N=63$ participants were recruited through Prolific, an online experimental platform, and paid \$15 for completing the study. To ensure quality and consistent data, we screened participants so that they could only complete the study on a desktop or laptop (excluding those on tablets and phones) and they were of United States nationality. Three participants were excluded from the analysis because they responded with either 30 or -30 when they rated their relative preference between the two gift cards on more than $80 \%$ of the trials. This indicated that they were not fully evaluating their preference, rather defaulting to the extreme values.

\section{Materials}

On each trial, a pair of gift cards to hypothetical local restaurants were presented. All participants saw the same set of 49 gift card pairs, but the order of presentation was randomized for each participant. The gift cards varied on 4 dimensions: dollar value (which ranged from $\$ 10-\$ 30$ ), restaurant rating (which ranged from 1-5 stars), average price per meal at the restaurant (which ranged from $\$ 5-\$ 20$ ), and distance from their home (which ranged from 0.1-10 miles). For the first response on each trial, participants specified their choice by either clicking a button labeled "Left"or a button labeled "Right". For the second response on each trial, participants recorded their relative preference between the two gift cards using a sliding scale from -30 to 30 .

\section{Procedure}

During the experiment, there were 7 blocks with 7 trials per block. At the beginning of a trial, a fixation cross was presented for two seconds. After that period, a pair of gift cards were presented and participants were prompted to either press the "Left" button to indicate that they prefer the left gift card or press the "Right" button to indicate that they would prefer the right gift card (for an example trial, see Figure 1).

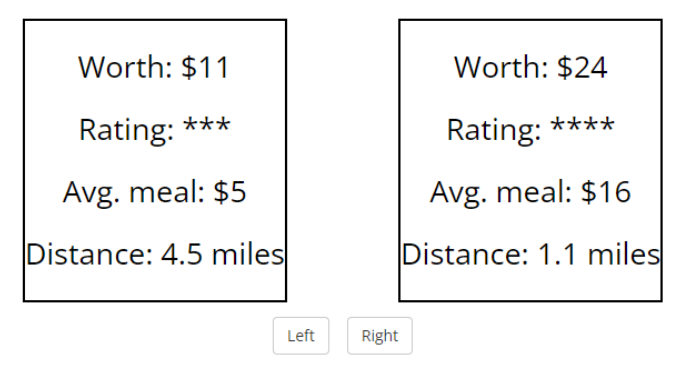

Figure 1: Example of a gift card pair with the responses.

After pressing the "Left" or "Right" button, the gift cards continued to be presented on the screen for a variable time interval of either $3,6,9,18,30$, or 45 seconds. Then, participants were asked to indicate their relative preference between the two gift cards using a slider on a scale of -30 to 30 , where a response of -30 indicated that they preferred the gift card on the left $\$ 30$ more than the one on the right and a response of 30 indicated that they prefer the gift card on the right $\$ 30$ more than the one on the left, and a response of 0 means they value them both equally. The second response was collected for an unrelated research question. At the end of the experiment, participants were asked what percentage of the time they paid attention to each attribute (on a scale of 0 to 100) to identify the importance they placed on each attribute.

\section{Model Formulation}

All three models outlined in this section are developed over an arbitrary number of preference states $(N)$, where the preference states are ordered according to preference strength for one alternative relative to the other. The $N$ preference states form an orthonormal basis for each model. We set $N=21$ when we fit the models to the experimental data, and the findings of the 21 preference state models are reported in the Results section. We chose to use 21 states because the model fits were worse with fewer states and adding more states beyond 21 did not improve fits. We did not use more than the necessary number of states needed to obtain good fits (21) because of the computational cost of the models.

\section{Markov Model}

We used a discrete-state, continuous-time Markov random walk process with absorbing boundaries and $N$ preferences states. The decision-maker's preference state $(\phi)$ is represented as a linear combination of the basis states (which are the $N$ preference states) such that the coefficient on each basis state represents the probability that the decision-maker's preference state is located in that basis state. Therefore, $\phi$ encodes a probability distribution over the basis states.

The dynamics of the Markov random walk process are governed by the generator matrix $(K)$. The generator matrix is a tridiagonal matrix that is specified using two free parameters: a drift rate $v_{M} \in(-\infty, \infty)$ which describes the rate at which the probability distribution moves toward one alternative relative 
to the other and a diffusion rate $\sigma_{M}^{2} \in(0, \infty)$ which describes the rate at which probability flows out of a basis state and into the adjacent states.

$$
K=\left[\begin{array}{ccccc}
-\beta & \alpha & 0 & \ldots & 0 \\
\beta & -\lambda & \ddots & \ddots & \vdots \\
0 & \beta & \ddots & \alpha & 0 \\
\vdots & \ddots & \ddots & -\lambda & \alpha \\
0 & \ldots & 0 & \beta & -\alpha
\end{array}\right]
$$

where $\alpha=\sigma_{M}^{2}-u \cdot v_{M}, \beta=\sigma_{M}^{2}+u \cdot v_{M}, \lambda=\alpha+\beta$, and $u \in$ $[-40,40]$ is the utility of the gift card pair, which is defined as the relative worth of one gift card to the other based on the four gift card attributes.

The dynamics of the Markov random walk are described by the Kolmogorov forward equation

$$
\frac{d}{d t} \phi=K \cdot \phi
$$

which defines how the probability distribution changes over time.

\section{Quantum Model}

We used a discrete-state, continuous-time quantum random walk process with absorbing boundaries and $N$ preference states. The decision-maker's preference state $(\psi)$ is represented as a linear combination of the basis states, similar to the Markov model. But, in the quantum model, the coefficient on each basis state represents the potential that the decision-maker's preference state is located in that basis state. Rather than operating on probabilities like the Markov random walk, the quantum random walk operates on probabilityamplitudes, or just amplitudes. Unlike probabilities, which are real numbers between 0 and 1 , amplitudes can be complex numbers whose magnitude is between 0 and 1 . The probability of the decision-maker's preference state being located in a given basis state is equal to the squared magnitude of the corresponding amplitude of that state. Therefore, $\psi$ encodes an amplitude distribution over the basis states.

The dynamics of the quantum random walk process are governed by the Hamiltonian matrix $(H)$. Similar to the generator matrix in the Markov model, the Hamiltonian matrix is a tridiagonal matrix that is specified using two free parameters: a drift rate $v_{Q} \in(-\infty, \infty)$ which is the slope (or constant force) of the potential function that determines the relative amount of amplitude flowing back into a basis state and a diffusion rate $\sigma_{Q}^{2} \in(0, \infty)$ which is the rate at which amplitude flows out of a basis state and into the adjacent ones. Note, the potential function does not necessarily need to be linear with a constant force like the one specified here. We just chose a linear potential function for simplicity.

$$
H=\left[\begin{array}{ccccc}
\mu_{1} & \sigma_{Q}^{2} & 0 & \ldots & 0 \\
\sigma_{Q}^{2} & \mu_{2} & \ddots & \ddots & \vdots \\
0 & \sigma_{Q}^{2} & \ddots & \sigma_{Q}^{2} & 0 \\
\vdots & \ddots & \ddots & \mu_{N-1} & \sigma_{Q}^{2} \\
0 & \ldots & 0 & \sigma_{Q}^{2} & \mu_{N}
\end{array}\right]
$$

where $\mu_{k}=u \cdot v_{Q} \cdot k, k$ is the index for state $k$, and $u$ is the same utility as in the Markov model.

The dynamics of the quantum random walk are described by the Schrödinger equation

$$
\frac{d}{d t} \psi=-i \cdot H \cdot \psi
$$

which defines how the amplitude distribution changes over time.

\section{Open System Model}

We used a discrete-state, continuous-time open system random walk process with absorbing boundaries and $N$ preference states. The decision-maker's preference state $(\rho)$ is represented using a density matrix, which is initialized as the outer product of the preference state in the quantum random walk $\rho=\psi \cdot \psi^{\dagger}$. The diagonal element in the $j$ row and column represents the probability that the decision-maker's preference state is located in that basis state.

Because the open system model is a hybrid of the Markov and quantum models, the dynamics of the open system random walk process are governed by both the generator matrix $(K)$ and the Hamiltonian matrix $(H)$. The generator matrix and Hamiltonian matrix take the same form as in the Markov and quantum models, respectively, so the dynamics of the open system model are specified using four free parameters: $v_{M} \in(-\infty, \infty), \sigma_{M}^{2} \in(0, \infty), v_{Q} \in(0, \infty)$, and $\sigma_{Q}^{2} \in(0, \infty)$.

The dynamics of the open system random walk are described by the quantum master equation

$$
\begin{aligned}
& \frac{d}{d t} \rho(t)=-i \cdot[H, \rho(t)] \\
& \quad+\sum_{j, k} \gamma_{j k} \cdot\left(\left(L_{j k} \cdot \rho(t) \cdot L_{j k}^{\dagger}\right)-0.5 \cdot\left\{\left(L_{j k}^{\dagger} \cdot L_{j k}\right), \rho(t)\right\}\right)
\end{aligned}
$$

where $\gamma_{j k}$ is equal to the element at row $j$ and column $k$ in the generator matrix $\left(\gamma_{j k}=K(j, k)\right)$ and $L_{j k}$ is a 21 by 21 matrix of 0 's, except at row $j$ and column $k$ there is a 1 . The first line of the right side of the quantum master equation ($i \cdot[H, \rho(t)])$ is the quantum component of the random walk, whereas the second line is the Markov component.

Note that when we define this equation, the two diffusion parameters, $\sigma_{M}^{2}$ and $\sigma_{Q}^{2}$, have similar interpretations. In the Markov model, $\sigma_{M}^{2}$ describes the rate at which probability flows out of a basis state and into the adjacent states; and in the quantum model, $\sigma_{Q}^{2}$ describes the rate at which amplitude flows out of a basis state and into the adjacent states. Since the 
open system model concurrently incorporates both Markov and quantum components, we believe that probability and amplitude should diffuse at the same rate $\left(\sigma^{2}=\sigma_{M}^{2}=\sigma_{Q}^{2}\right)$.

To control the relative influence of the Markov and quantum components on the open system random walk, we added a weighting parameter, $\omega \in[0,1]$, to the quantum master equation. With the addition of the weighting parameter, we can reparameterize the master equation, still in terms of four free parameters $\left(\omega, v_{M}, v_{Q}\right.$, and $\left.\sigma^{2}\right)$

$$
\begin{aligned}
& \frac{d}{d t} \rho(t)=(1-\omega) \cdot(-i \cdot[H, \rho(t)]) \\
&+\omega \cdot\left(\sum_{j, k} \gamma_{j k} \cdot\left(\left(L_{j k} \cdot \rho(t) \cdot L_{j k}^{\dagger}\right)-0.5 \cdot\left\{\left(L_{j k}^{\dagger} \cdot L_{j k}\right), \rho(t)\right\}\right)\right)
\end{aligned}
$$

where now $H$ is defined using $v_{Q}$ as the force and $\sigma^{2}$ as the diffusion rate and $K$ (which is where the $\gamma_{j k}$ is taken from) is defined using $v_{M}$ as the drift rate and $\sigma^{2}$ as the diffusion rate. Note that when $\omega=0$, the open system model reduces to the quantum random walk; and when $\omega=1$, the open system model reduces to the Markov random walk.

\section{Other Parameters}

Non-decision Time To account for the time included in the total response time that was not dedicated to the decision process (e.g., motor response), we included a non-decision time parameter in the models, $T_{N D}$.

Discrete to Continuous Response Time Distribution We began by formulating each model as a continuous-time, discrete-state random walk process with absorbing boundaries. The absorbing boundaries in each model are the two terminal basis states. If the preference state is measured to be in the first basis state, then the random walk process ends and the decision-maker selects the left gift card. Similarly, if the preference state is measured to be in the 21 st basis state, then the random walk process ends and the decision-maker selects the right gift card. To implement absorbing boundaries, each model must repeatedly evolve the preference state for some amount of time $(\tau)$ and then measure the probability that the state is in either of the terminal states. The evolution and subsequent measurement of the preference state is referred to as a step in the random walk. The probability that the preference state is in either of the terminal states on step $n$ is equal to the probability that the random walk process ends after $n$ steps (and the probability that the response time $t$ is given by $t=\tau \cdot n+T_{N D}$ ). The number of steps must be discrete, so the response time distribution is discrete as well.

Although the current state-of-the-art 2AFC model, the drift-diffusion model, is a continuous-time, continuous-state model (which allows it to have a continuous response time distribution), we could not directly compare the quantum or open system model to the drift-diffusion model because there is no continuous-time, continuous-state quantum random walk. To work around this, we assumed that each step in the random walk is exponentially distributed with rate $g$, rather than deterministically occurring at a fixed interval. The rate $g \in(0, \infty)$ is fit as a free parameter. By assuming the measurements are exponential distributed (described by a continuous probability density) rather than occurring at deterministic times as specified by a constant time interval, the response time distribution of each random walk is continuous rather than discrete.

\section{Results}

We excluded trials with response times larger than $20 \mathrm{~s}$ to exclude trials where participants were likely not attending to the task.

\section{Model Comparison}

The parameters for both the Markov and open system models were fit using maximum likelihood estimation. The likelihood of each model was converted to the $G^{2}$ statistic to perform model comparison $\left(G^{2}=-2 \cdot L L\right.$, where $L L$ is the log-likelihood of the model given the data and the set of parameters). Since the Markov and quantum models are nested models of the open system model, we can perform a $\chi^{2}$ test to determine whether we should reject the simpler models in favor of the more complex open system model. The mean $G^{2}$ value for each model across participants, and the number of parameters used in each model, are displayed in Table 1

Table 1: Comparison of mean fits across participants.

\begin{tabular}{|c|c|c|}
\hline Model & Mean $G^{2}$ & $k$ \\
\hline \hline Markov & 222.69 & 4 \\
\hline Quantum & 238.23 & 4 \\
\hline Open system & 217.82 & 6 \\
\hline
\end{tabular}

Comparing the mean fits of each model, $\Delta G_{M}^{2}=222.69-$ $217.82=4.87, \Delta G_{Q}^{2}=238.23-217.82=20.41$, and $\Delta k=$ $6-4=2$, where $k$ is the number of free parameters, $\chi^{2}\left(\Delta G_{M}^{2}=4.87, \Delta k=2\right)$ results in $p=0.087$ and $\chi^{2}\left(\Delta G_{Q}^{2}=\right.$ $20.41, \Delta k=2)$ results in $p=3.7 \cdot 10^{-5}$, so we do not have enough evidence on average to reject the Markov model in favor of the more complex the open system model but we do have enough evidence on average to reject the quantum model in favor of the open system model.

When looking at the individual participants using the same $\chi^{2}$ test, we found that for 16 out of the 60 participants, there was enough evidence to reject the Markov model in favor of the open system model; and for all 60 participants, there was enough evidence to reject the quantum model in favor of the open system model.

\section{Predicted Response Time Distributions}

As an alternative to quantitatively distinguishing between the different models by comparing their fits, observing each model's predicted response time distribution can illuminate qualitative differences between the models. For example, the response time distribution of a quantum random walk can be 
multimodal, whereas that of a Markov random walk must be unimodal Busemeyer et al. (2006). Figure 2 illustrates the predicted response time distributions using the best-fit parameters for participant 51 and utility $=1$ for all three models.

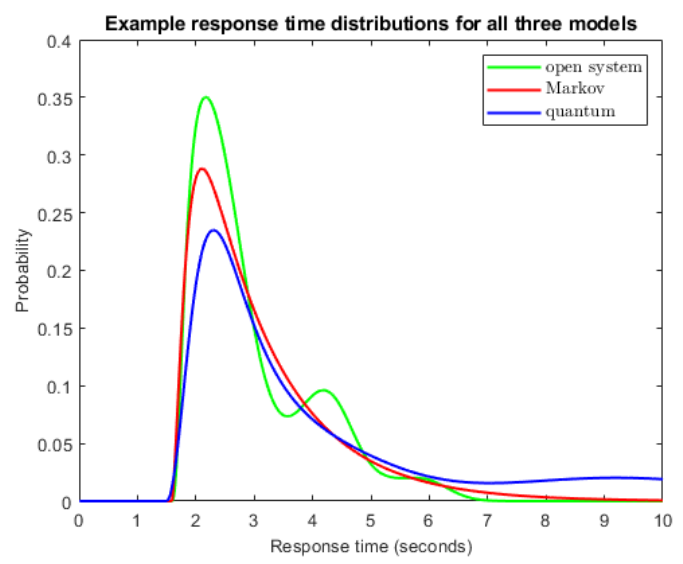

Figure 2: Response time distributions using the best-fit parameters for participant 51 and utility $=1$.

Figure 2 demonstrates that the open system model can predict multimodal response time distributions like the quantum model. But, looking at the tails of each distribution, the tail of the Markov and open system models are much smaller compared to that of the quantum model. As a hybrid of the Markov and quantum models, it appears as though the open system models combined the advantageous features of the two models to more accurately account for the observed response times.

\section{Weighting Parameter}

The weighting parameter is a key component of the open system model, as it controls the relative influence that the Markov and quantum components have on the open system (Busemeyer, Kvam, \& Pleskac, 2020).

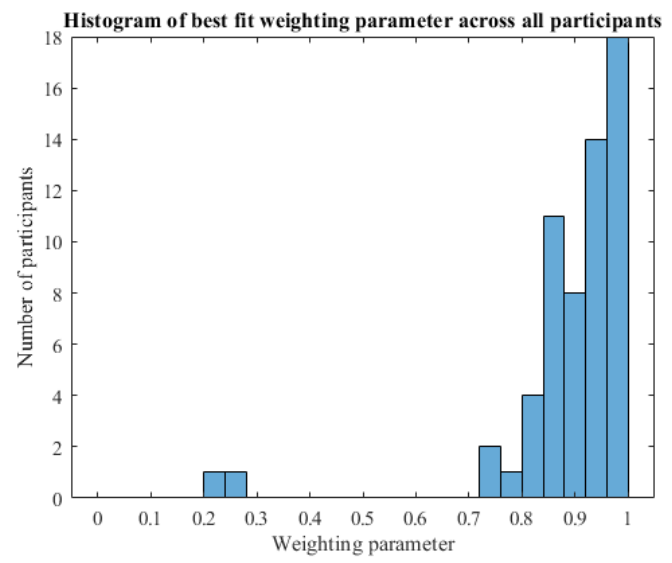

Figure 3: Histogram of the best fit weighting parameter across the 60 participants.
As illustrated in Figure 3, most of the weighting parameters pile up around 0.9, which suggests that the Markov component of the open system model has a stronger influence on the random walk compared to the quantum component. Although, this finding could be due to the fitting program getting stuck in local minima when optimizing the parameters of the open system model. When fitting the model, we used the parameters of the Markov model as an initial guess. It would make sense that, given the complexity of the open system model's parameter space, the optimization algorithm would keep the weighting parameter near 1 , as the initial parameters are sure to give a decent fit (equal to the Markov) when the weighting parameter is 1 . The two sets of best fit parameters with the weighting parameter between 0.2 and 0.3 could provide a basis to further explore sets of starting parameters.

\section{Discussion}

When accounting for behavior during decision-making tasks, both Markov and quantum random walk models have their respective strengths. The Markov model excels at modeling pairwise choice and response time (Busemeyer et al., 2006), whereas the quantum model excels at modeling pairwise choice and confidence/preference strength and naturally predicts both interference effects and oscillations in preference strength (Kvam et al., 2015, 2021). Being a hybrid of the two, the open system model has the potential to combine the best aspects of both models.

The potential of the open system model has already been realized when predicting preference strength (Kvam et al., 2021), and here, for the first time, we extended that model to choice and response time. Although on average, we cannot reject the Markov model in favor of the open system model, we can reject the Markov model in favor of the open system model for 16 out of the 60 participants. Given that modeling choice and response time is the biggest strength of the Markov model, the success of the open system model is greatly encouraging and warrants further investigation. Plus, the open system model significantly outperformed the quantum model on all 60 participants, which demonstrates its improvement over the pure quantum model.

Going forward, we plan to examine each model's ability to predict pairwise choice, response time, and preference strength data. Since the open system model notably outperformed the Markov and quantum models when fit to mean preference strength, and nearly outperformed the Markov model when predicting pairwise choice and response time, we are confident the open system model will outperform the Markov and quantum models when predicting pairwise choice, response time, and preference strength.

\section{Acknowledgments}

This material is based upon work supported by the Air Force Office of Scientific Research under award number FA955020-0027. 


\section{References}

Bhattacharya, R. N., \& Waymire, E. C. (2009). Stochastic processes with applications. SIAM.

Bruza, P., Wang, Z., \& Busemeyer, J. R. (2015). Quantum cognition: a new theoretical approach to psychology. Trends in cognitive sciences, 19(7), 383-393.

Busemeyer, J. R., Gluth, S., Rieskamp, J., \& Turner, B. M. (2019). Cognitive and neural bases of multi-attribute, multi-alternative, value-based decisions. Trends in cognitive sciences, 23(3), 251-263.

Busemeyer, J. R., Kvam, P., \& Pleskac, T. (2020). Comparison of markov versus quantum dynamical models of human decision making. Wiley Interdisciplinary Reviews: Cognitive Science, 11(4), e1526.

Busemeyer, J. R., Wang, Z., \& Townsend, J. (2006). Quantum dynamics of human decision-making. Journal of Mathematical Psychology, 50(3), 220-241.

Busemeyer, J. R., Zhang, Q., Balakrishnan, S., \& Wang, Z. (2020). Application of quantum-markov open system models to human cognition and decision. Entropy, 22(9), 990.

Fuss, I. G., \& Navarro, D. J. (2013). Open parallel cooperative and competitive decision processes: A potential provenance for quantum probability decision models. Topics in Cognitive Science, 5(4), 818-843.

Hughes, R. (1989). The structure and interpretation of quantum mechanics. Harvard university press.

Kolmogorov, A. (1950). Foundations of the theory of probability.

Kvam, P., Busemeyer, J. R., \& Pleskac, T. (2021). Temporal oscillations in preference strength provide evidence for an open system model of constructed preference. Scientific reports, 11(1), 1-15.

Kvam, P., Pleskac, T., Yu, S., \& Busemeyer, J. R. (2015). Interference effects of choice on confidence: Quantum characteristics of evidence accumulation. Proceedings of the National Academy of Sciences, 112(34), 10645-10650.

Martínez-Martínez, I., \& Sánchez-Burillo, E. (2016). Quantum stochastic walks on networks for decision-making. Scientific reports, 6(1), 1-13.

Ratcliff, R., Smith, P. L., Brown, S. D., \& McKoon, G. (2016). Diffusion decision model: Current issues and history. Trends in cognitive sciences, 20(4), 260-281.

Wang, Z., \& Busemeyer, J. R. (2016). Comparing quantum versus markov random walk models of judgements measured by rating scales. Philosophical Transactions of the Royal Society A: Mathematical, Physical and Engineering Sciences, 374(2058), 20150098.

Yearsley, J. M. (2017). Advanced tools and concepts for quantum cognition: A tutorial. Journal of Mathematical Psychology, 78, 24-39. 